\title{
Goal Formulation based on Communicative Principles
}

\author{
Kristiina Jokinen* \\ Computational Linguistics Laboratory \\ Graduate School of Information Science \\ Nara Institute of Science and Technology \\ 8916-5 Takayama, Ikoma, Nara \\ 630-01 JAPAN \\ kris@is.aist-nara.ac.jp
}

\begin{abstract}
The paper presents the Constructive Dialogue Model as a new approach to formulate system goals in intelligent dialogue systems. The departure point is in general communicative principles which constrain cooperative and coherent communication. Dialogue participants are engaged in a cooperative task whereby a model of the joint purpose is constructed. Contributions are planned as reactions to the changing context, and no dialogue grammar is needed. Also speech act classification is abandoned, in favour of contextual reasoning and rationality considerations.
\end{abstract}

\section{Introduction}

Two general approaches can be distinguished in dialogue management: the structural approach, which uses a dialogue grammar to capture regularities of the dialogue in terms of exchanges and moves (Bilange, 1992; Cawsey, 1993; Grosz and Sidner, 1986), and the intention-based approach, which classifies the speaker's beliefs and intentions into speech acts, and uses planning operators to describe them (Appelt, 1985; Allen and Perrault, 1980; Bunt et al., 1984). Both regard natural language as purposeful behaviour, but differ in how this behaviour is to be described. The former sees dialogues as products and compiles participants' beliefs and intentions into a predefined dialogue structure, whereas the latter focusses on the participants' goals, and hides the structure in the relations between acts which contain appropriately chosen sets of beliefs and intentions as their preconditions and effects.

We will not go into detailed evaluation of the approaches, see e.g. (Jokinen, 1994), but draw attention to three aspects of dialogues which have

${ }^{*}$ I am grateful to Yuji Matsumoto for providing an excellent research environment during my JSPS Postdoctoral Fellowship, and Graham Wilcock for helpful discussions. not been properly addressed before, although widely acknowledged in literature, and important in building robust Natural Language interfaces:

1. dialogue is a collaborative process and its structure is recognised by external observation, not prescribed as an internal constraint of dialogue management (Sacks et al., 1974; Clark and Wilkes-Gibbs, 1990),

2. the speakers' beliefs and intentions in a given dialogue situation are various, and cannot all be checked when deciding on the next response (Cherniak, 1986),

3. communicative acts are part of social activity, constrained by normative obligations of rational agency (Allwood, 1976).

We discuss these aspects from the point of view of cooperative goal formulation and present the Constructive Dialogue Model as a new approach to plan system responses. Our departure point is in general communicative principles which constrain cooperative and coherent communication, and radical steps are taken in two respects: the dialogue grammar is abandoned as an infeasible way to describe dialogues, and also speech act recognition is abandoned as a redundant labelling of intention configurations. The first step means that the structure is not built according to structuring rules, but emerges from local coherence as the dialogue goes on. The second step means that beliefs and intentions are dealt with by reasoning about the utterance context and communicative constraints instead of speech act types. The decision about what to say next falls out as a result of the agent complying with the communicative principles which refer to the agent's rationality, sincerity, motivation and consideration. Combined with contextual knowledge, they account for the acceptability of different alternative responses.

The paper is organised as follows. The theoretical framework and its formalisation as the Constructive Dialogue Model are discussed in Section 2. Section 3 presents how the system's communicative goal is determined, and Section 4 provides comparision with related work. Finally, conclusions and future directions are given in Section 5 . 


\section{Constructive Dialogue Model}

\subsection{Rational, cooperative way to react}

Rational agents try to follow the principles of Ideal Cooperation (Allwood, 1976) in communication: (1) assume a joint purpose, (2) show cognitive consideration (epistemic rationality regarding appropriate ways to react) and ethical consideration (intention to react in a way that does not prevent the partner from fulfilling her goals), and (3) trust. that the partner is acting according to the same principles. Ideal cooperation does not mean that the agents always react in the way the partiner in tended to evoke, but rather, it sets the normality assumptions for the way the agents would behave if no disturbing factors were present. As (Gal liers, 1989) points out, conflict resolution forms an important part of human communication, and if systems are always ready to adopt the user's role, they become rigid and unrealistic. However, if the conflict becomes so serious that it makes any cooperation impossible, communication will break down as well. Rational agents thus try to communicate so as to conform to the shared assumptions about operationally appropriate and ethically acceptable acts in a given situation (Jokinen, 1995).

Fmpirical dialogue research has emphasised collaborative nature of dialogues (Sacks et al., 1974; Clark and Wilkes-Gibbs, 1990). Also computationally oriented dialogue studies show that the users express themselves vaguely and continue with follow-up questions (Moore and Paris, 1993), and our corpus ${ }^{1}$ supports the view that even simple information seeking dialogues resemble negotiations rather than straightforward question-answer sequences. Based on these obeservations and the theoretical assumptions of Ideal Cooperation, we distinguished the main factors in rational, cooperative dialogue management as follows: whercas the information seeker is not expected to teach the information provider how to look for the information. The roles can be further differentiated with respect to social factors such as acquaintance of the addressee and formality of the situation.

- Communicative obligations. Social, normative requirements that concern the agent's sincerity (exchange information which is true or for which evidence can be provided), motivation (exchange information which is related to one's goals and strategies), and consideration (exchange information which the partiner is able to deal with).

- Task. Gives rise to communication. 'Task goals are planned to complete a real world task (rent a car, book a flight, repair a pump) but because of uneven distribution of knowledge, the agents usually need to collaborate to achieve the goal, and thus formulate communicative goals to obtain missing information, cf. (Guinn, 1994).

\section{B. Communicative act:}

- Expressive and evocative attitudes. To distinguish between the effects of an utterance and the intentions behind it, Austin's concept of illocution is split up into two: $e x$ pression of the speaker's attitude and evocation of a reaction in the partner; perlocution corresponds to what is actually achieved by the act: the evoked response, cf. (Allwood, 1976). Expression may differ from evocation (irony, indirectness), and the evoked response from the evocative intentions (the agent requests information that the partner cannot or does not want to disclose; the agent fails to frighten the partner because this has guessed the agent's malicious intentions).

\begin{tabular}{|c|c|c|}
\hline Surface form & Expressive intentions & Evocative intentions \\
\hline Declarative & express a belief: & share the belief: \\
\hline & want (Sp, know (He,P)) & want $\left(\mathrm{Sp}\right.$, want $\left(\mathrm{H}_{\theta}\right.$, know $\left.\left.\left(\mathrm{He}_{\mathrm{e}} \mathrm{P}\right)\right)\right)$ \\
\hline Inter rogative & $\begin{array}{l}\text { desire for intormation: } \\
\text { want }(S p, k n o w(S p, P))\end{array}$ & $\begin{array}{l}\text { provicle the desired intormation: } \\
\text { want }\left(\mathrm{Sp}, \operatorname{want}\left(\mathrm{He}_{e}, \text { know }(\mathrm{Sp}, \mathrm{P})\right)\right)\end{array}$ \\
\hline Imperative & desire for action: & (provide) action: \\
\hline & $\operatorname{want}\left(\mathrm{Sp}, \mathrm{do}\left(\mathrm{H}_{\theta}, \mathrm{P}\right)\right)$ & $\operatorname{want}\left(\mathrm{Sp}, \operatorname{want}\left(\mathrm{He}_{e}, \mathrm{do}\left(\mathrm{He}_{\Theta}, \mathrm{P}\right)\right)\right)$ \\
\hline Exclamative & express action: & attend to the action: \\
\hline & $\operatorname{want}(S p, d o(S p, P))$ & $\operatorname{want}\left(\mathrm{Sp}, \operatorname{want}\left(\mathrm{H}_{\theta}, \mathrm{do}(\mathrm{Sp}, \mathrm{p})\right)\right)$ \\
\hline
\end{tabular}

Figure 1: Conventional association of expressive and evocative intentions with surface form, modified from (Allwood, 1992).

\section{A. Communicative situation:}

- Role. Characterised by global communicative rights and obligations of the agents. E.g the information provider is expected to give information which is relevant for the task,

\footnotetext{
${ }^{1}$ The corpus was collected by the Wizard-of- $\mathrm{O} z$ technique with users trying to find information on carhire companies and restaurants in a particular area, and is reported in (Nivre, 1992).
}

\section{Communicative context:}

- Expectations. Fvocative intentions put pressure on the agent to react in a particular way. Conventional expectations, carried by the surface form (Fig. 1), serve as anchoring points in reasoning about the partner's communicative goal (Cohen and Levesque, 1990).

- Initiatives. If the agent has initiated a communicative goal, she "has the initiative" and 
also the right to pursue the goal until it is achieved or not relevant anymore. She also has the right to expect the partner to collaborate or at least not prevent the agent from achieving her goal.

- Unfulfilled goals. If the expressive attitudes of the partner's response match the evocative intentions of the agent's contribution, the communicative goal of the agent's contribution is fulfilled. An unfulfilled goal is pushed forward or stored for later processing. When the agent has the right to take the initiative, a previously unfulfilled goal can be taken up. If the goal is still unfulfilled and relevant, it is resumed, otherwise dropped.

- Thematic coherence. A competent agent relates the topic of her contribution to what has been discussed previously or marks an awkward topic shift appropriately; otherwise the agent risks being understood. Thematic relatedness is based on the types of relationships which occur in the domain.

\subsection{The CDM System}

The theoretical framework is formalised as an approach to dialogue management called the Constructive Dialogue Model, CDM (Jokinen, 1994). In CDM, the dialogue is an instrument to exchange new information on a particular topic to complete a real world task, and it is managed locally by reacting to the changed dialogue context.

The task division and information flow in a CDM system ${ }^{2}$ is shown in Fig. 2. The dialogue manager operates on the Context Model which is a dynamic knowledge base containing facts about the agents' goals, expressive and evocative attitudes, central concepts (topic), and new information. It also has access to three static knowledge bases: Communicative Principles (knowledge about rational, cooperative communication), Application Model (knowledge about tasks and roles), and World Model (general knowledge about the entities and their relations in the world). ${ }^{3}$

Dialogue contributions are constructed in three phases corresponding to the three main processing tasks. Analysis of the input message results in the user's communicative goal, and contains four subtasks: determine the explicitness level, interpret the propositional content, check coherence and verify obligations. Evaluation of the user goal concerns an appropriate joint purpose and determines the next system goal. Response specifies the system's communicative goal up to the semantic representation using the same subtasks as analysis but in a reverse order. Evaluation and response form the agent's reaction.

\footnotetext{
${ }^{2}$ The prototype is implemented in SICStus Prolog 2.1 , running under UNIX ${ }^{T M}$ on a Sun SPARCStation.

${ }^{3}$ Linguistic knowledge is encoded in a linguistic lexicon and grammar, and not discussed here.
}

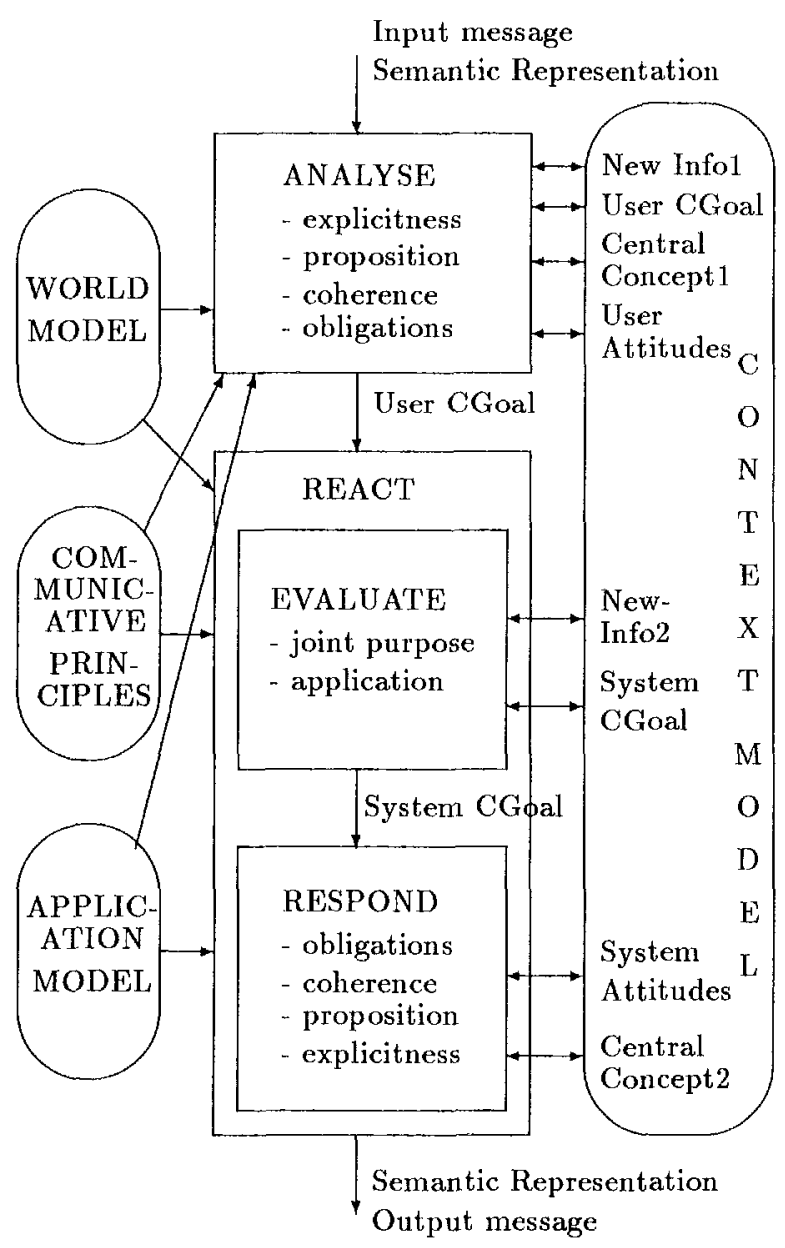

Figure 2: Information flow in the CDM system.

The Context Model is represented as a partitioned Prolog database and the predicates have an extra argument referring to the contribution whose processing introduced them. In the attitude language the predicates know, want and do represent belief, intention and action, respectively, $s$ refers to the system and $u$ to the user. Communicative Principles are reasoning rules of the form:

$$
\begin{aligned}
& \text { if cntxtFact } 1, \ldots, \operatorname{cntxtFact} N \\
& \text { then cntxtFactM+1, ., cntxtFactK. }
\end{aligned}
$$

The World Model uses neo-Davidsonian event representation, and the Application Model provides mappings from World Model concepts to task and role related facts.

\section{Cooperative Goal Formulation}

In CDM, joint purpose represents the communicative strategy that an agent has chosen in a particular situation to collaborate with her partner. It is determined by evaluating the partner's goal with respect to the communicative context: expectations, initiatives, unfulfilled goals and coherence. Assigning binary values to these aspects, we get $2^{4}=16$ joint purposes, summarised in Fig. 3 . The 


\begin{tabular}{|l|l|l||l|l||}
\hline goals & initiative & central concept & expected response & non-expected response \\
\hline \multirow{3}{*}{ fulfilled } & \multirow{2}{*}{ speaker } & related & finish/start & continue/start \\
\cline { 3 - 5 } & & unrelated & finish/specify & object/specify \\
\cline { 2 - 5 } & partner & related & follow-up-new & somethingelse \\
\cline { 2 - 5 } & & unrelated & new-request & new-indir-request \\
\hline \multirow{3}{*}{ unfulfilled } & speaker & related & backto & subquestion \\
\cline { 2 - 5 } & & unrelated & repeat-new & object \\
\cline { 2 - 5 } & partiler & related & follow-up-old & continue \\
\cline { 3 - 5 } & & unrelated & new-question & notrelated \\
\hline
\end{tabular}

Figure 3: Possible joint purposes if the contextual factors are assigned binary values.

reasoning rules are as follows (examples of the alternatives can be found in (Jokinen, 1994)):

1. The agent has fulfilled goals only, and the initiative: Finish the dialogue or start a new one depending on the pending task goals (finish/start/continue/object/specify). Maintain the initiative if the response is related, give the initiative if unrelated.

2. The agent has fulfilled goals only, but no initiative: Adopt the partner's goal. Maintain the initiative if the response is expected (follow-up-new, new-request), take the initiative if the response is non-expected (somethingelse, new-indir-request).

3. The agent has unfulfilled goals, and the initiative: Adopt the partner's goal if the response is thematically related (backto, subquestion), persist with the own goal if unrelated (repeat-new, object). Maintain the initiative if the response is expected, give the initiative if non-expected.

4. The agent has unfulfilled goals, but no initiative: Adopt the partner's goal. Maintain the initiative if the response is thematically related (follow-up-old, continue), take the initiative if unrelated (newquestion, notrelated).

The joint purpose describes communicative intentions in a context where no speaker obligations or considerations hold. In order to attend the requirements of a particular communicative situation, the joint purpose needs to be specified with respect to the agent's role, task and communicative obligations.

Specification of the joint purpose via the Application Model captures the cognitive consider. ation of Ideal Cooperation: the agent plans her response to be operationally appropriate in the current situation. The result is a communicative goal (c-goal), a set of communicative intentions instantiated according to the current task and role.

The c-goal is then filtered through communicative obligations which implement the ethical consideration of Ideal Cooperation: the agent's communicative competence shows in the ways she can realise the same c-goal in various situations. Some communicative obligations are listed in Fig. 4.
Sincerity: "do I know this or can provide evidence?"

1. Wverything that the speaker asserts or implies is true unless otherwise explicitly stated.

Motivation: "can I say this?"

1. Everything that the speaker wants to know or wants the partner to do is motivated except if the speaker cannot take the initiative on it.

2. Hverything that addresses what the partner wanted to know or wanted the speaker to do is motivated, except if the speaker cannot disclose the information or do the act.

3. Fverything that is related to $\mathrm{CC}$ is motivated if not already known.

4. Everything that informs of inconsistency is motivated if not already known.

Consideration: "may I say this?"

1. If the partner's goal cannot be fulfilled (presuppositions are false, facts contradictory, no information exists), it is considerate to inform why (explain, compensate, initiate repair).

2 . If the response would repeat previous information, it is considerate to leave this implicit unless the information is assigned a special emphasis.

3 . If the partner's response is unrelated, it is considerate to inform of the irrelevance, given that the speaker has unfulfilled goals.

4. If the partner did not request a piece of related information, it is considerate to include this explicitly in the response, given that the speaker intends to close the topic.

Figure 4: Some communicative obligations.

\subsection{Example}

Consider the following sample dialogue where the system's task is to provide service information to the user:

U1: I need a car.

S1: Do you want to buy or rent one?

U2: Rent.

S2: Where?

U3: In Bolton.

S3: OK. Here are the car hire companies in Bolton: ....

The analysis of the first user contribution $U 1$ is given in Fig. 5. The content of the user's c-goal is inferred from the World Model which says that 'needing a car' can be interpreted as 'wanting to have a car'. 


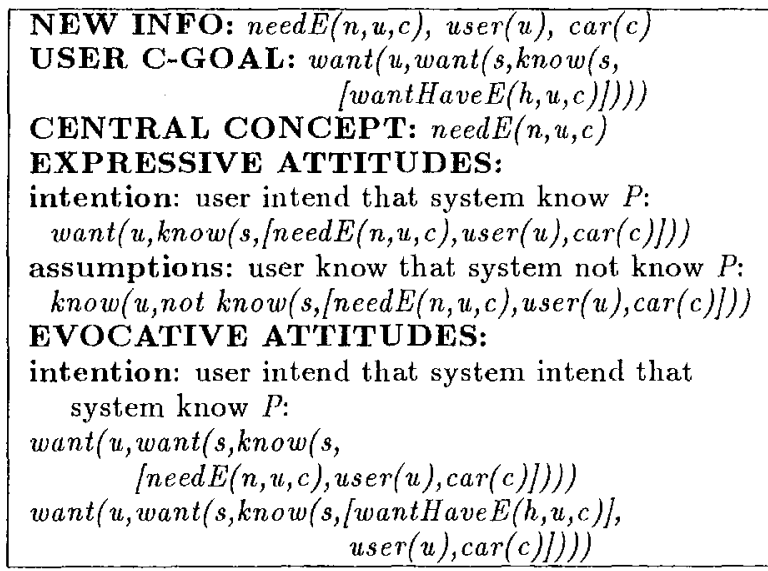

Figure 5: Context Model after the user contribution $I$ need a car. The constants $n, u, c, h$ identify instantiated concepts.

In the beginning of the dialogue the system has no unfulfilled goals, and its role as an obedient information provider does not allow it to have the initiative. Moreover, any contribution is trivially unrelated to the previous topic, since no previous topic exists. According to the Joint Purpose rule (2), the user's c-goal is thus adopted, and the system also takes the initiative, since the user contribution is non-expected (an information seeker is expected to start with a question or a request). The joint purpose becomes new-indir-request with "user wants to have a car" as the content, i.e. the communicative strategy is to share the user's want to have a car, and check if this want can be satisfied within the Application Model.

The system cannot provide the user with a car, but it can provide information about the services that enable the user to have a car. Application Model lists car hire companies and car garages as possible services, so the communicative goal is formulated as to know which is the preferred service. The services are associated with renting or buying cars, thus the disjunction is realised as $S t$.

The system responses $S 2$ and $S 3$ are based on the same strategy backto: the system 'goes back' to adopt the user's previous unfulfilled goal and tries to satisfy this in the updated context. ${ }^{4}$ However, they carry different c-goals due to different specification in the Application Model: S2 aims at narrowing down the database search, $S 3 \mathrm{com}$ pletes the original task. Finally, the communicative obligation Consideration (4) requires that the application service (car hire company) and location (Bolton) are explicitly expressed in $S 3$ before the list of services.

\footnotetext{
${ }^{4}$ The user response fulfills expectations and is thematically related, and the system has the initiative and unfulfilled goals, at least one based on the original task to provide information.
}

\section{Discussion and related work}

In Section 1 we pointed out three important aspects of dialogues which have been insufficiently accounted for in the earlier approaches to dialogue management. In CDM, these aspects form the basis of the system's functionality: dialogues are regarded as collaborative activities, planned locally in the changed context as reactions to the previous contributions and governed by the rationality principles of Ideal Cooperation. The logical omniscience assumption is tackled by partitioning the Context Model and focussing on specific knowledge with the help of thematic coherence; also rationality considerations constrain reasoning.

$\mathrm{By}$ adhering to general communicative principles, CDM provides a new and uniform way to treat various phenomena that have been separately studied in previous research: goal formulation, coherence and cooperativeness. Communicative principles function on the following levels:

1. Determination of the joint purpose: reasoning about a communicative strategy in the context (expectations, initiatives, unfulfilled goals, thematic coherence)

2. Selection of the communicative goal: filtering the joint purpose with respect to the agent's role and task.

3. Realisation of the goal: specifying the goal in regard to the communicative obligations sincerity, motivation and consideration.

However, we also use insights from the huge body of research that exists on dialogue management and natural language planning. For instance, the negotiative nature of dialogues is emphasised in (Moore and Paris, 1993) who show how rhetorical knowledge can be combined with the knowledge about the speaker's intentions and communicative goals so that the system can understand follow-up questions or justify its explanations. Our work differs from this in that we study general requirements of communication rather than rhetorical relations and their augmentation with speaker intentions, to determine appropriate responses. It is possible to modify our joint purpose algorithm with information about rhetorical relations so as to check expectations in regard to argumentation, or to include rhetorical knowledge in the obligations used when reasoning about multisentential contributions, but as our primary goal has been to specify communicative principles and use them in the formalisation of the cooperative and rational nature of dialogues, this kind of extension is left for future.

(Guinn, 1994) presents a model of mixedinitative negotiation as collaborative problem solving. His Missing Axiom approach demonstrates collaboration and communication between two agents who possess complementary knowledge: if the agent's information is not sufficient to allow 
completion of the proof the agent is set to do, the agent attempts to provide the missing axioms through interaction. This is similar to our basic assumption of how domain tasks give rise to communication. The differences lie again in our emphasis on 'Rational and Cooperative Communication' as opposed to 'Interaction as a Failure to Prove'.

In abandoning dialogue grammar and speech act classification, we agree with the conmon view currently held among researches: dialogue structure is constructed on the basis of the participants' beliefs and intentions, and speech act types are at most convenient abbreviations for a set of attitudes held by the speakers, but do not constitute an explanation of the dialogue ( $\mathrm{Co}_{-}$ hen and Levesque, 1990; Galliers, 1989). We also use contextual knowledge extensively, and connect intention-based approaches to practical dialogue management: rationality and cooperation are not only tied to the agent's beliefs and intentions of the desidered next state of the world, but also to the wider social context in which the communication takes place.

\section{Conclusion and future directions}

This paper has presented a new way to formulate system goals in intelligent dialogue systems. It advocates a view-point where the system's functionality is improved by relating the dialogue situation to communication in general. The constraints of rational, cooperative communication provide the framework in which to deal with contributions: communicators have a joint purpose, they obey communicative obligations and they trust that the partner behaves so that these constraints are fulfilled. Dialogues are dynamic constructions, and contributions are locally planned and realised so that the communicative requirements of the dialogue as a whole are respected.

Current interests concern the extension of the communicative principles into different activities and agent roles. This contributes to the generality of the model by spelling out specific requirements of different communicative situations. It also enables us to study strategic planning and how different roles affect the obligations that the agents want to obey (e.g. in conflict situations). Work is now in progress to cover other types of task dialogues, and to enhance the implementation.

\section{References}

J. F. Allen and C. R. Perrault. 1980. Analyzing intention in utterances. Artificial Intelligence, 15:143-178.

J. Allwood. 1976. Linguistic Communication as Action and Cooperation. Department of Linguistics, University of Göteborg. Gothenburg Monographs in Linguistics 2.
J. Allwood. 1992. On dialogue cohesion. Technical Report Gothenburg Papers in T'heoretical Linguistics 65, University of Gothenburg.

D. Appelt. 1985. Planning Natural Language Utterances. Cambridge University Press, Cambridge.

E. Bilange. 1992 Dialogue personnemachine. Modélisation et réalisation informatique. Hermès, Paris.

H. C. Bunt, R. J. Beun, F. J. H. Dols, J. A. van der Linden, and G. O. thoe Schwartzenberg. 1984. The TENDUM dialogue system and its theoretical basis. T'echnical Report 19, IPO.

A. Cawsey. 1993. Explanation and Interaction. The Computer Generation of Explanatory Dialogues. The MIT Press, Cambridge, MA.

C. Cherniak. 1986. Minimal Rationality. The MI'T Press. Cambridge, MA.

H. H. Clark and D. Wilkes-Gibbs. 1990. Referring as a collaborative process. In P. R. Cohen, J. Morgan, and M. F. Pollack, editors, Intentions In Communication, pages 463-493. The MLI' Press. Cambridge, MA.

P. R. Cohen and H. J. Levesque. 1990. Rational interaction as the basis for communication. In P. R. Cohen, J. Morgan, and M. E. Pollack, editors, Intentions in Communication, pages 221. 255. The MIT Press. Cambridge, MA.

J. R. Galliers. 1989. A theoretical framework for computer models of cooperative dialogue, acknowledging multi-agent conflict. Technical Report 172 , University of Cambridge.

B. J. Grosz and C. L. Sidner. 1986. Attention, intentions, and the structure of discourse. Computational Linguistics, 12(3):175-204.

C. I. Guinn, 1994. Meta-Dialogue Behaviors: Improving the Efficiency of Human-Machine Dialogue. A Computational Model of Variable Initiative and Negotiation in Collaborative Problem-Solving. Ph.D. thesis, Duke University.

K. Jokinen. 1994. Response Planning in Informa tion-Seeking Dialogues. Ph.D. thesis, UMISI'.

K. Jokinen. 1995. Rational agency. In M. Fehling, editor, Rational Agency: Concepts, Theories, Models, and Applications, pages 89-93. Proceedings of The AAAI-95 Fall Symposium.

J. D. Moore and C. L. Paris. 1993. Planning text for advisory dialogues: Capturing intentional and rhetorical information. Computational Linguistics, 19(4):651-694.

J. Nivre. (Ed.)(1992). Corpus collection and analysis. Technical Report D2.1, PLUS deliverable.

II. Sacks, E. A. Schegloff, and G. Jefferson. 1974. A simplest systematics for the organization of turn-taking in conversation. Language, 50(4):696-735. 